\title{
Modelling and observation of transionospheric propagation results from ISIS II in preparation for ePOP
}

\author{
R. G. Gillies ${ }^{1}$, G. C. Hussey ${ }^{1}$, H. G. James ${ }^{2}$, G. J. Sofko ${ }^{1}$, and D. André ${ }^{1}$ \\ ${ }^{1}$ Institute for Space and Atmospheric Studies, University of Saskatchewan, Saskatoon, Saskatchewan, Canada \\ ${ }^{2}$ Communications Research Centre, Ottawa, Ontario, Canada
}

Received: 11 September 2006 - Revised: 4 January 2007 - Accepted: 16 January 2007 - Published: 1 February 2007

\begin{abstract}
The enhanced Polar Outflow Probe (ePOP) is scheduled to be launched as part of the Cascade Demonstrator Small-Sat and Ionospheric Polar Explorer (CASSIOPE) satellite in early 2008. A Radio Receiver Instrument (RRI) on ePOP will receive HF transmissions from various groundbased transmitters. In preparation for the ePOP mission, data from a similar transionospheric experiment performed by the International Satellites for Ionospheric Studies (ISIS) II satellite has been studied. Prominent features in the received 9.303-MHz signal were periodic Faraday fading of signal intensity at rates up to $13 \mathrm{~Hz}$ and a time of arrival delay between the $\mathrm{O}$ - and $\mathrm{X}$-modes of up to $0.8 \mathrm{~ms}$. Both features occurred when the satellite was above or south of the Ottawa transmitter. Ionospheric models for ray tracing were constructed using both International Reference Ionosphere (IRI) profiles and local peak electron density values from ISIS ionograms. Values for fade rate and differential mode delay were computed and compared to the values observed in the ISIS II data. The computed values showed very good agreement to the observed values of both received signal parameters when the topside sounding $f o \mathrm{~F} 2$ values were used to scale IRI profiles, but not when strictly modelled IRI profiles were used. It was determined that the primary modifier of the received signal parameters was the $f o \mathrm{~F} 2$ density and not the shape of the profile. This dependence was due to refraction, at the $9.303-\mathrm{MHz}$ signal frequency, causing the rays to travel larger distances near the peak density where essentially all the mode splitting occurred. This study should assist in interpretation of ePOP RRI data when they are available.
\end{abstract}

Keywords. Radio science (Ionospheric physics; Radio wave propagation) - Space plasma physics (Numerical simulation studies)

Correspondence to: R. G. Gillies

(rgg646@mail.usask.ca)

\section{Introduction}

In early 2008, the Cascade Demonstrator Small-Sat and Ionospheric Polar Explorer (CASSIOPE) small satellite is scheduled to be launched (Yau et al., 2002, 2006). The satellite bus, as well as many of the instruments, are Canadian designed and built. The satellite will consist of two payloads, a commercial prototype data storage and forward system called Cascade and a scientific package of instruments called the enhanced Polar Outflow Probe (ePOP). The main scientific goals of ePOP are to study ion outflows from the polar region, radio wave propagation, and ionospheric tomography (Yau et al., 2006). The main instruments used for the radio wave propagation experiment will be ground-based transmitters, such as Super Dual Auroral Radar Network (SuperDARN) HF radars (Greenwald et al., 1995) and Canadian Advanced Digital Ionosondes (CADI) (MacDougall et al., 1995), and the Radio Receiver Instrument (RRI) (James, 2003, 2006a, b; James et al., 2006; Wang et al., 2004) on the ePOP payload.

The RRI consists only of a radio receiver (i.e., a passive system) and four 3-m antennas. Each antenna has an independent receiving channel, but the monopoles will often be operated as two perpendicular 6-m dipole antennas. The ground-based transmitters will radiate HF radio waves. These will propagate through the ionosphere, and the RRI instrument will have the ability to observe various parameters of these arriving waves including their polarization state.

Along the wave propagation path ionospheric electron density enhancements and/or depletions (blobs, patches, troughs, etc.) are expected to modify the received signal parameters. As a result, it is expected that the signal recorded by the RRI will have a complex structure. As an initial step toward interpretation of the signal received by the RRI when it is flown the influence of various ionospheric structures along the propagation path must be assessed. This paper deals with preliminary modelling research which has been performed to achieve this goal. Once the effects of

Published by Copernicus GmbH on behalf of the European Geosciences Union. 
ionospheric properties upon the signal are understood it is envisioned that the received signal can be used to measure or at least detect these ionospheric structures.

At this point, no data exist for the SuperDARN-RRI experiment and thus only modelling predictions can be studied. Therefore, it was of interest to examine past transionospheric experiments to provide a first step to deal with SuperDARNRRI simulations. Examples of such experiments are the transionospheric portions of the International Satellites for Ionospheric Studies (ISIS) satellite missions from the 1970s (Franklin and Maclean, 1969). To this end, data from the ISIS II mission during 1978 have been studied in detail.

\section{The ISIS II transionospheric experiment}

\subsection{Background}

The ISIS II satellite (Daniels, 1971) was launched in 1971 into an essentially polar orbit (89 inclination) at $1400 \mathrm{~km}$ altitude. The primary instrument on the satellite was a radio sounder consisting of 18-m and 73-m dipole antennas and an HF transmitter and receiver. This instrument was mainly configured for topside soundings of the ionosphere, but for special experiments it was configured to receive signals from ground-based transmitters. For several ISIS II passes over a transmitter located in Ottawa, Canada (Geographic coordinates: $45.42^{\circ} \mathrm{N}, 75.70^{\circ} \mathrm{W}$; Geomagnetic coordinates: $56.36^{\circ} \mathrm{N}, 0.53^{\circ} \mathrm{E}$ ), the topside sounder alternated between a swept-frequency ionosonde mode and a fixed-frequency receiver mode. In the ionosonde mode, the satellite would perform a sweep for $\sim 20 \mathrm{~s}$. Then the sweep would turn off and the signal from a fixed-frequency ground-based transmitter would be received for $\sim 25 \mathrm{~s}$. This switching occurred throughout a pass over a given transmitter. At the altitude of the satellite, this resulted in a topside scan for approximately $1.2^{\circ}$ latitude of satellite travel and then transionospheric data for approximately $1.3^{\circ}$ latitude of satellite travel.

The Ottawa transmitter used in this experiment radiated a power of $1 \mathrm{~kW}$ at (usually) $9.303 \mathrm{MHz}$. Other frequencies were used but are not examined in this study. The antennas of the transmitter consisted of two perpendicular dipoles oriented horizontal to the Earth. The transmitting sequence consisted of square wave pulses sent at a repetition frequency of $180 \mathrm{~Hz}$.

For the ISIS II observations presented in this study, passes were selected based on three criteria. First, orbital data of ISIS II had to be available for a particular pass. Second, it was necessary for the data from a pass to have been digitized (Bilitza et al., 2004). The digitized data from these passes were made available by the Alouette-ISIS Data Restoration project of the U.S. National Space Science Data Center (NSSDC, 2004). Finally, a pass was only examined if ground transmitted signal had been received for a significant portion of the pass. The most probable reason for a pass not meeting the third criterion was that the foF2 frequency was higher than or comparable to the transmitted frequency of $9.303 \mathrm{MHz}$, with the result that the ground-based transmitted waves were reflected back to the Earth. On this basis, a detailed examination was made of eighteen passes from June and July 1978. All of these passes occurred between either 01:00-04:00 UT ( 20:00-23:00 LT (local evening)) or 13:00-16:00 UT ( 08:00-11:00 LT (local morning)). Finally, it should be noted that all the data were from quiet or slightly disturbed geomagnetic conditions (typical $K_{p}$ values were between two and three or less). Therefore it was not unreasonable to assume, and the analysis to be presented supports this, that the ionosphere was relatively stable and smoothly varied in both altitude and latitude under these conditions. This is especially true for the ISIS II study wherein the analyzed data was predominantly from directly above or to the south of the Ottawa transmitter. Of course, the ePOP mission is focused on the polar (and auroral) region(s) where the ionosphere can be very active and disturbed geomagnetically. However, the results from the slightly disturbed events presented in this paper will serve as a reference with which to compare the dynamic variations expected during more disturbed periods.

\subsection{General signal characteristics}

In general, the signal received by ISIS II behaved as expected. The signal was weaker and noisier at satellite locations closest to the Ottawa-transmitter horizon. Directly above the transmitter, the signal was strongest. When the satellite was north of the transmitter, periodic fading of signal intensity at a rate of $0.1 \mathrm{~Hz}$ was observed. This fading was caused by the spacecraft spin at a rate of $3 \mathrm{rpm}$. In the southern portions of the pass, faster periodic fading of signal intensity was observed due to Faraday rotation of the wave as will be discussed in Sect. 2.4. Satellite spin fading should be observed south of the transmitter as well because the dipole receiving antenna was rotating with respect to an incoming linear wave. However, this spin fading was not detectable. The reason is that the Faraday rotation effect and the spinning of the dipole antenna both contribute to the observed fade rate. Since the Faraday fading was typically much higher than $0.1 \mathrm{~Hz}$, it has been assumed that the observed fade rate is effectively only due to Faraday rotation of the wave and the satellite spin has been neglected in the southern portions of the pass. Due to this coupling of satellite spin and the Faraday rotation effect, $0.1 \mathrm{~Hz}$ should be added to or subtracted from the measured Faraday fade values, but since it is unknown which way the satellite was spinning relative to the Faraday rotation and $0.1 \mathrm{~Hz}$ is quite small compared to the typical fade rates, this error has been neglected. Also apparent in southern portions of the passes was a time delay between the arrival of the two modes of propagation at the satellite. This differential mode delay is discussed in the next section. 


\subsection{Differential mode delay}

When an electromagnetic wave propagates in a magnetoionic medium there exist two modes of propagation, the ordinary mode (O-mode) and the extraordinary mode (X-mode) (Budden, 1961). These modes propagate with different indices of refraction and thus different propagation paths and group speeds result. The different propagation properties of the two modes caused the received signal pulses at ISIS II to be split into separate mode components. This splitting is evidenced as a time delay between the arrival of the O-mode and Xmode at the receiver. An example of differential mode delay is in plot a) in Fig. 1. Differential mode delay is observed at the beginning (O-mode only band above the combined (and dominant) $\mathrm{O}$ - and $\mathrm{X}$-mode band) and end (X-mode only band below the combined (and dominant) $\mathrm{O}$ - and $\mathrm{X}$-mode band) of each pulse as shown in the top plot in Fig. 1.

The locations and magnitudes of differential mode delay instances were determined directly from observations of the received signal data. The resolution of the available data resulted in an error of $\pm 0.1 \mathrm{~ms}$ in all differential mode delay values. There was measurable differential mode delay in fifteen of the eighteen studied passes and it was mostly observed when the satellite was to the south of the Ottawa transmitter. The magnitude of differential mode delay ranged from $0.1 \mathrm{~ms}$ to $0.8 \mathrm{~ms}$. The magnitude was, in general, larger for latitudes farther from the transmitter.

It should be noted that there is some differential mode attenuation due to collisions in the D- and E-regions as discussed with reference to Fig. 10 from James et al. (2006), which showed that the differential absorption between the modes at $90 \mathrm{~km}$ is $\sim 0.15 \mathrm{~dB} / \mathrm{km}$ and drops to less than $0.02 \mathrm{~dB} / \mathrm{km}$ at $100 \mathrm{~km}$. It was therefore expected that the differential attenuation between the modes would have been on the order of one $\mathrm{dB}$ at most. A disturbed ionosphere may induce more collisions at these altitudes, but since all the passes examined here were for quiet conditions, the effect of absorption on the received signal parameters was not considered significant and is not considered in the present study.

\subsection{Periodic Faraday fading}

The second regularly observed feature of the transionospheric propagation in the ISIS II data was periodic fading of signal intensity. Periodic fading of a single mode (signal intensity of only either the O-mode or X-mode wave periodically varies) is illustrated in Fig. 1 as cross-section c) for the O-mode only band. The single mode fading is difficult to discern in the top plot, but clearly discernable in crosssection line plot c). Single mode fades are believed to be the result of focusing and defocusing of single mode waves by ionospheric enhancements and depletions acting like lenses. Discussion of single mode fades can be found in James et al. (2006) and is not further considered here.
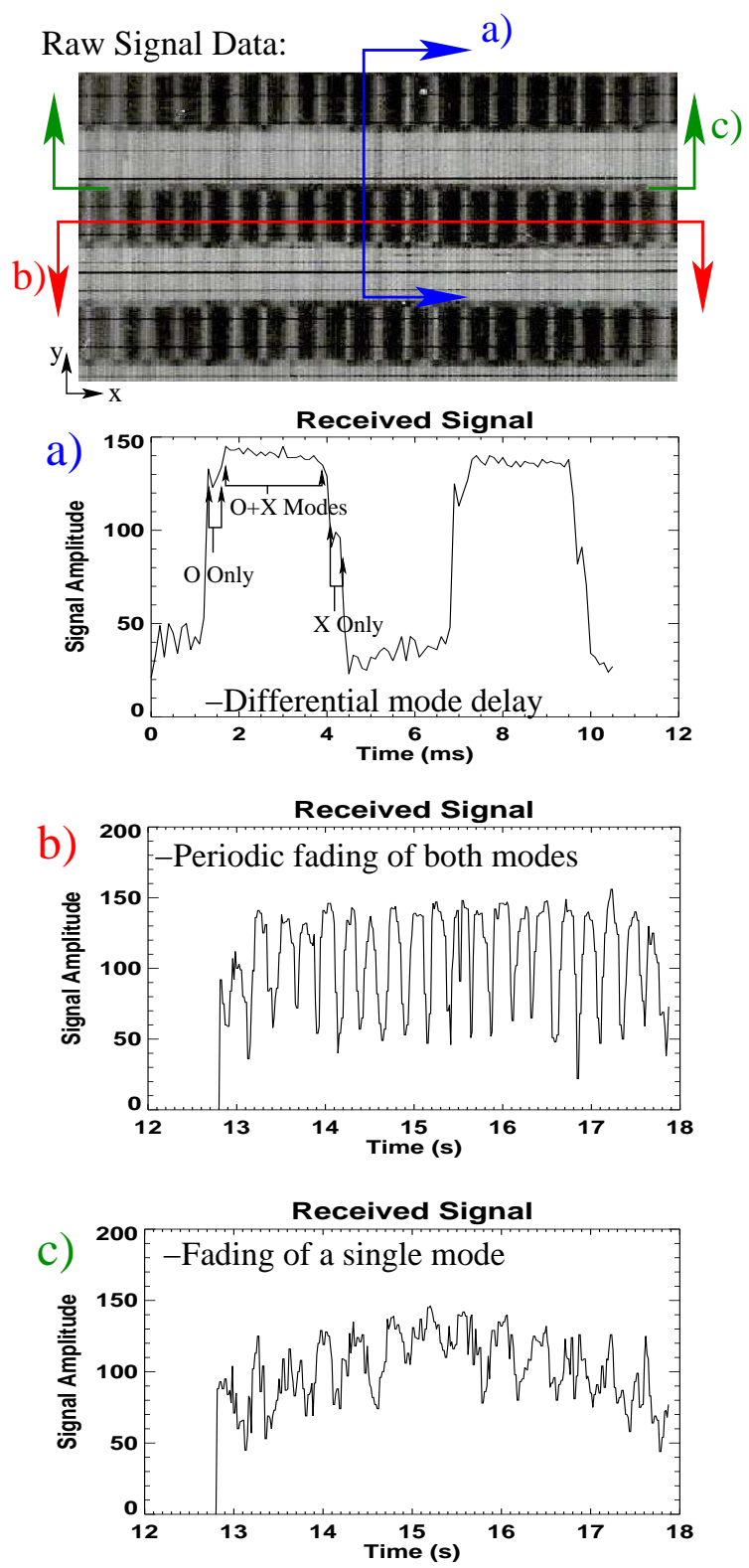

Fig. 1. A six second example of signal received by the ISIS II satellite at 13:59:12 UT, 3 July 1978. Dark on the grey-scale data plot at the top represents received signal amplitude on the dipole antenna. Individual amplitude scans are plotted as a function of time in the $-y$-direction. The grey-scale plot results from the juxtaposition of many successive amplitude scans in the $+x$-direction. Individual $180-\mathrm{Hz}$ pulses are seen as three dark horizontal bands. This pattern is maintained over several seconds of time in the x-direction. Examples of: (a) Differential mode delay is observable at the beginning and end of each pulse. (b) Periodic Faraday fading occurs in portions of signal with both modes at a rate of $4.0 \mathrm{~Hz}$. (c) Single mode fades are also present. Note, the y-axis for plots (a), (b), and (c) has arbitrary relative amplitude units. 
An example of periodic fading of signal intensity when both modes were received at the same time is illustrated in cross-section b). These fading instances occurred for up to several tens of seconds at a time. A likely explanation for this periodic fading is the Faraday rotation of the linearly polarized total electric field of the transmitted wave. Since the antenna is a linear dipole, strong signal was received when the electric field was nearly aligned with the dipole axis. As the satellite moved along its orbit the incoming linear wave would Faraday rotate out of alignment with the dipole axis. Weakest signal occurred when the antenna dipole and electric field were closest to perpendicular. This continuous changing of the electric field orientation led to a periodic fading of signal intensity as the satellite travelled through space.

The periodic fading of both modes was observed in eight of the eighteen studied passes. Periodic fading was analyzed by performing a Fourier analysis on the sections of data where signal was received by ISIS II from the Ottawa ground transmitter. The fading rates ranged from $0.5 \mathrm{~Hz}$ to $13 \mathrm{~Hz}$. As with differential mode delay, periodic fading was observed only south of the transmitter and the fade rate frequency was observed to increase in magnitude at locations more equatorward of the transmitter.

\section{Transionospheric modelling}

\subsection{3-D ray tracing program}

The Appleton-Hartree equation for the index of refraction of a radio wave in a magnetoionic medium was used to derive computational ray tracing equations based on the ray formalism of Haselgrove (1963). Applied to the magnetoionic refractive index, the Haselgrove (1963) equations provide both the group velocity and rate of change in wave vector (in three dimensions) given the wave-vector direction and wave frequency and a set of propagation conditions such as magnetic field strength and direction, and electron density. The authors have applied these equations in a computer program for full three-dimensional ray tracing. The program calculates the path, at a given frequency, that either the $\mathrm{O}$ - or $\mathrm{X}$-mode travels from a fixed transmitter to a satellite position. The program uses the International Geomagnetic Reference Field (IGRF) model (Maus et al., 2005) for determining the magnetic field and takes a user supplied one- or two-dimensional electron density profile. Using a Newton iteration method (James, 2006a), the ray trace converges to intersect with the location of the satellite within $10 \mathrm{~m}$ in typically a few iterations. It should be pointed out that the 10-m convergence criterion, in practice, applies to a line perpendicular to the propagation path at the satellite and corresponds to an error along the ray path of at most $10 \mathrm{~cm}$. Hence, this corresponds to a very small phase-path difference between the modes at HF frequencies (Gillies, 2006).

\subsection{Ray trace modelling for ISIS II}

As discussed in Sect. 2.2, eighteen passes of the ISIS II satellite over the Ottawa transmitter were examined in detail. These passes produced Faraday fading and differential mode delay in the received signal. Ray tracing computations have been performed for these passes. Comparisons between the Faraday fading and differential mode delay values obtained from the ray tracing computations and the actual observed data were then made. In order to predict values for Faraday fade rates and differential mode delays, the ray tracing program found a ray path for every $0.1^{\circ}$ latitude of the satellite orbit over the transmitter. Initially, the International Reference Ionosphere (IRI) model (Bilitza, 2001) was utilized to obtain electron density profiles. The resulting Faraday fade rates and differential mode delay values that were obtained by using the IRI model did not show good agreement with the observed values, although overall gross trends were exhibited. This is illustrated in Fig. 2 for Faraday fade rate and Fig. 3 for differential mode delay. These figures present the observed ISIS II values of the two quantities (as dashed lines with diamonds indicating data points) and the predicted values using the IRI generated profile (as dash-dotted lines) for the afternoon pass on 8 July. Notice that the observed data are at latitudes south of the Ottawa $\left(45.4^{\circ} \mathrm{N}\right)$ transmitter while the ray tracing results demonstrate that Faraday fading and differential mode delay should be observed north of the transmitter as well. This discrepancy will be discussed in Sect. 4.1. Both observed and predicted values of fade rate and differential mode delay show an increase with increasing distance from the transmitter, but the predicted values increase at a much faster rate than the observed values.

Recall that the ISIS II satellite performed a topside ionogram scan roughly once every $2.5^{\circ}$ in latitude. Therefore, it was decided to utilize the $f o \mathrm{~F} 2$ values that the satellite sounder had determined to develop more realistic twodimensional electron density profiles. These simulated profiles utilized the $f_{o} \mathrm{~F} 2$ values taken directly from the topside scans while relying on the IRI model for the shape of the profiles (i.e., the foF2 values were used to scale IRI profiles). It should be noted that this scaling option did not simply multiply the entire profile by the scaling factor. In the IRI model the various altitude regions use different ionospheric models to determine electron density so a change (scaling) of the $f_{o} \mathrm{~F} 2$ value, for example, only affects electron densities near it. Figure 4 shows the $f_{o} \mathrm{~F} 2$ density values that were obtained from both the IRI and the topside soundings. On this day the IRI overestimates the peak values by typically $\sim 30 \%$. Scaling to the foF2 values led to a significant improvement in agreement between the computed and observed values. There are some limitations to how accurately the profiles represented the actual ionospheric conditions. First, the sounder performed a scan only every $2.5^{\circ}$ latitude or roughly every $350 \mathrm{~km}$. This separation of measurements was insufficient to resolve small scale ionospheric structures. Secondly, 


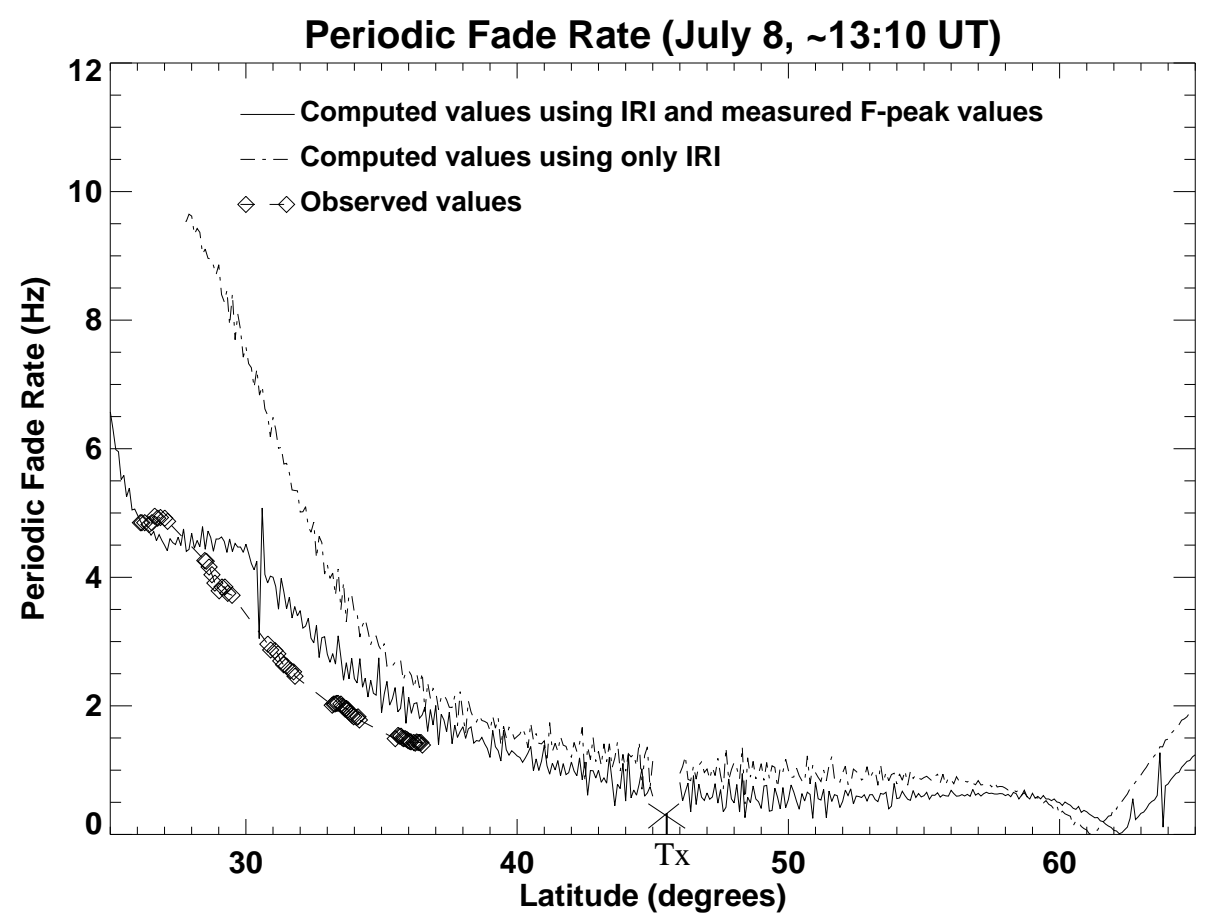

Fig. 2. An example comparison between simulated and observed Faraday fade rates. This pass occurred on 8 July 1978, 13:10 UT. The two computed curves were obtained using two different electron density profiles, one generated using only the IRI model and the other using the model scaled to the topside sounding $f o \mathrm{~F} 2$ values.

the determination of an accurate $f o \mathrm{~F} 2$ value was not always possible due to noise and/or weak signal near the cut-off frequency in some of the ionograms. At times this resulted in ambiguity in the peak plasma frequency by up to $1 \mathrm{MHz}$. Finally, there was error introduced from the fact that only the F-region peak was used to adjust the IRI profile. This meant that the shape of the profile was not the same as the actual profile during the experiment. However, as will be shown in Sect. 4.3, the shape of the profile mattered little provided the peak density was reasonably accurate.

Using the $f_{o} \mathrm{~F} 2$ value to scale the IRI model electron density profiles resulted in much better agreement between actual data and the computed fade rates and differential mode delays, as shown in Figs. 2 and 3. It can be seen that the computed results using the topside ionogram peaks are much closer to the observed values than the computed values using only the IRI. This comparison demonstrates the necessity of reliable $f o \mathrm{~F} 2$ values for the ray tracing.

\section{Discussion}

\subsection{ISIS II transionospheric analysis}

Examination of ISIS II data has revealed two prominent features in the observed signal received during the transionospheric experiment. The first is a measurable time delay be- tween the arrival at the satellite receiver of the two modes of propagation. The second is periodic fading in the received signal, explained as Faraday fading arising when a linearly polarized wave electric field is incident on a dipole receiving antenna.

The differential mode delay values ranged from 0.1 to $0.8 \mathrm{~ms}$ and were observed at latitudes to the south of the Ottawa transmitter. The transmitted frequency was $9.303 \mathrm{MHz}$ for the ISIS experiment considered here. For future experiments of this type, such as SuperDARN-ePOP collaborations, the transmitter will be able to operate at frequencies from about $8 \mathrm{MHz}$ to $20 \mathrm{MHz}$. Hence, depending on the operating frequency, the ePOP RRI should observe differential mode delay values comparable to the ISIS receiver. The most likely reason for greater differential mode delay observed to the south of the transmitter is that the overall ionospheric electron density increases due to the increase in photoionization with decreasing latitude. This has the effect of bringing the transmitted wave frequency closer to the cutoff frequencies of the modes. The consequence is that the $\mathrm{X}$-mode, which is closer in frequency to its cutoff frequency than the $\mathrm{O}$-mode, experiences more dispersion and greater signal delay.

Periodic Faraday fading of the two modes occurred at rates up to $13 \mathrm{~Hz}$ and, as with mode delay, was observed only south of the transmitter. An explanation for this is slightly 


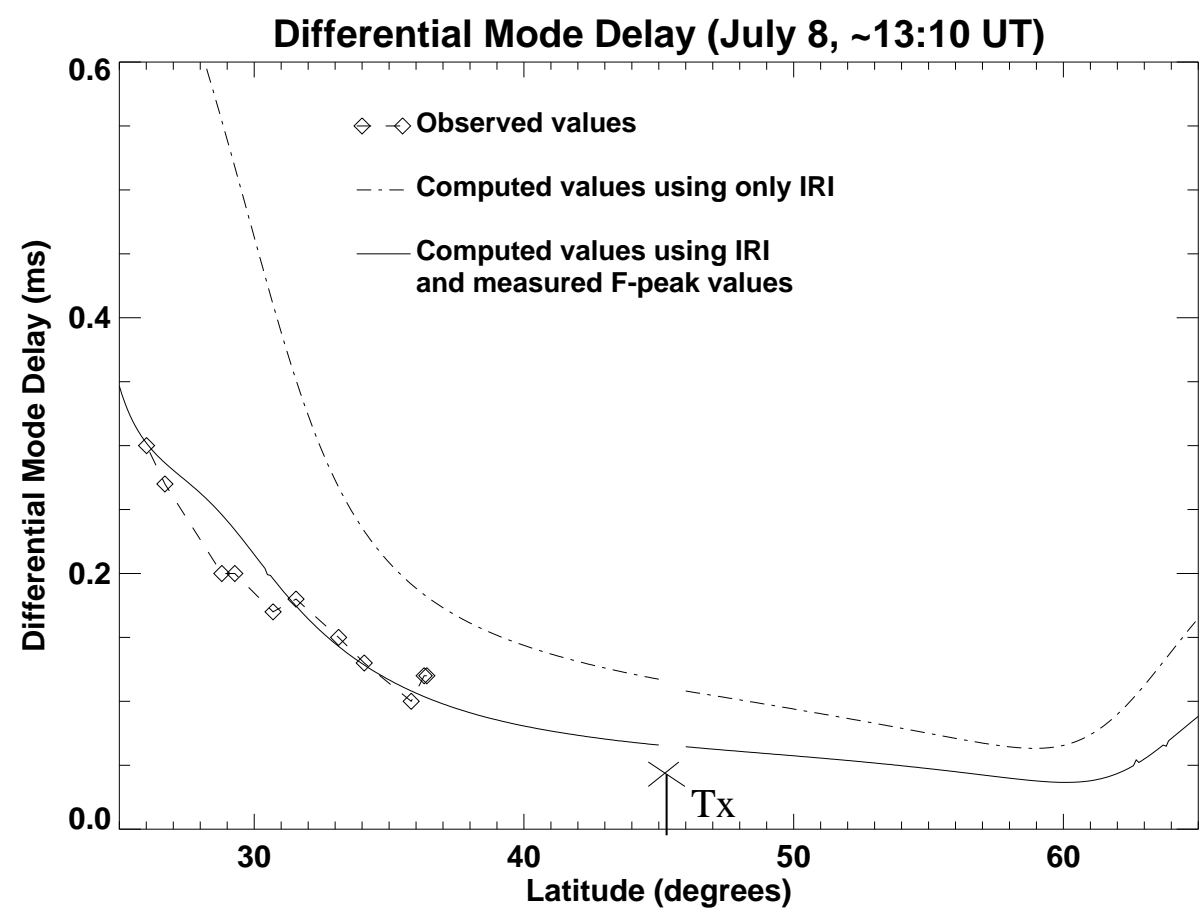

Fig. 3. An example comparison between simulated and observed differential mode delay times. This pass occurred on 8 July 1978, 13:10 UT. The two simulated curves represent two different electron density profiles, one generated using only the IRI model and the other using the model scaled to the topside sounding foF2 values.

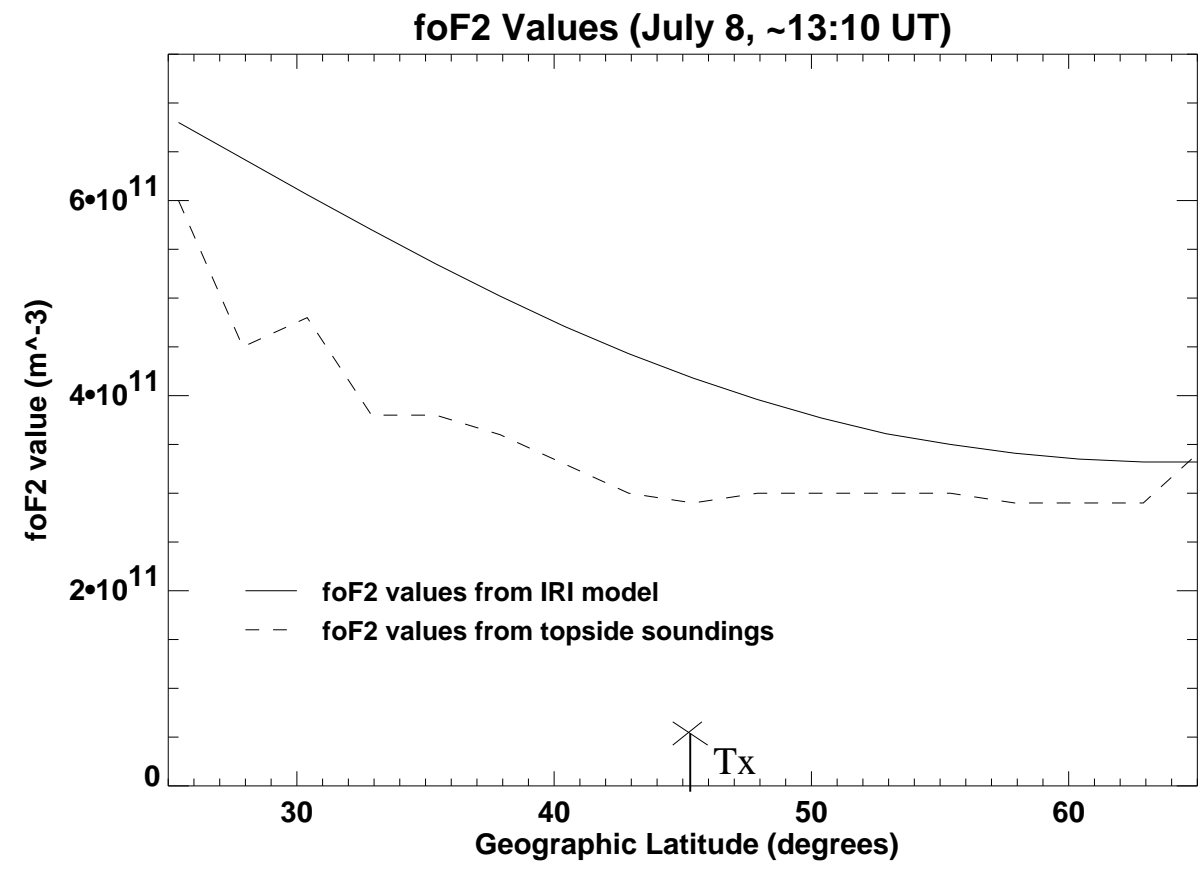

Fig. 4. The $f o F 2$ values obtained from both the IRI model and the ISIS II topside soundings for the pass on 8 July 1978, 13:10 UT. 


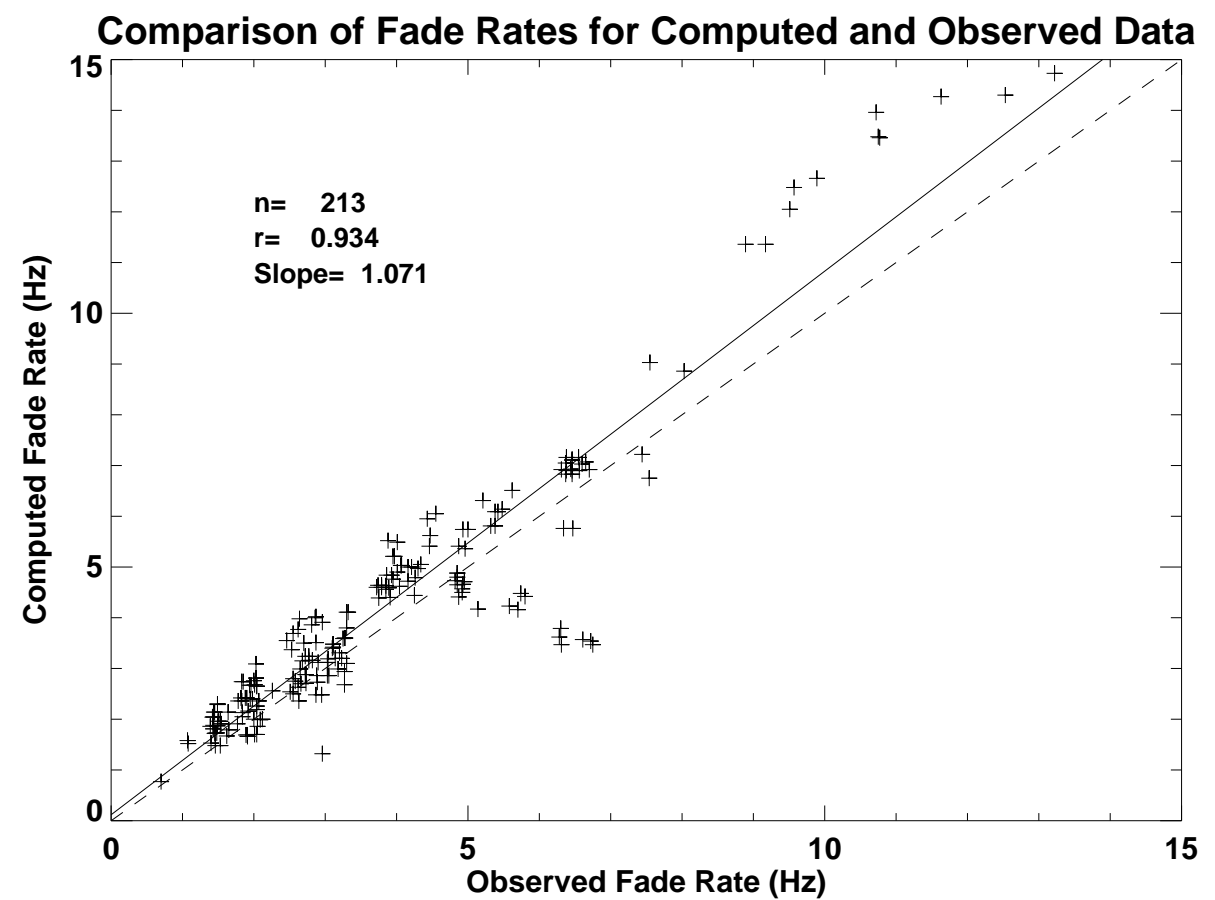

Fig. 5. A linear regression comparison of all computed and observed Faraday fade rates for the ISIS II experiment. The dashed line is the ideal fit and the solid line is the best fit. The regression analysis used 213 points and gave a regression coefficient of 0.934 and a slope of 1.07 .

more complex than that for differential mode delay. The most probable reason for the lack of observable Faraday fading north of the transmitter was a disparity between the received power of the $\mathrm{O}$ - and $\mathrm{X}$-modes. As presented by James (2006a) and James et al. (2006), in several instances the Xmode was at least $10 \mathrm{~dB}$ stronger than the $\mathrm{O}$-mode. This amplitude disparity was clearly not due to absorption in the E-region because the X-mode is absorbed more than the $\mathrm{O}$ mode. This disparity was possibly due to the distribution of power between the $\mathrm{O}$ - and $\mathrm{X}$-modes when the wave propagated in a northward direction. This is currently under investigation.

This 10-dB disparity had the effect of preventing Faraday rotation (which requires circularly polarized modes of roughly equal amplitude) during portions of a pass. Instead, this disparity in mode amplitude would have caused the combined wave to not be strictly linearly polarized and thus the received signal polarization state would have been complex. This complexity could not be properly analyzed with only a single dipole antenna. The RRI on ePOP will be a useful instrument for analyzing the polarization state of the signal received poleward of the transmitter as the antenna will consist of two crossed dipoles.

Another minor factor that would not be supportive of Faraday rotation occurring north of the transmitter was the angle between the propagation vector and magnetic field direction (the aspect angle) becoming close to perpendicular (i.e., the propagation conditions are not as favourable for the Faraday rotation effect). However, Fig. 8 of James et al. (2006), which takes this factor into account, indicates that deep Faraday fades are expected throughout a pass, and clearly this is not observed in the ISIS II data.

\subsection{Observed and modelled ISIS II signal comparisons}

Comparisons between observed and simulated differential mode delay and periodic fading values have been performed for each simulated pass. Figures 2 and 3 demonstrated that the IRI model alone cannot develop electron density profiles that are sufficiently realistic to reproduce these received signal parameters. However, given a relatively quiet ionosphere ( $K_{p}$ less than four for this data set), the use of measured foF2 densities to scale IRI profiles produces better agreement between the ray tracings and the observations. A disturbed ionosphere would probably have many smaller scale structures that topside soundings taken every $2.5^{\circ}$ in latitude would not resolve and this might significantly lower the agreement. Also, a disturbed ionosphere would result in higher collision frequencies and differential absorption in the D- and E-region portion of the transionospheric wave path.

In order to quantify the comparisons of all the passes, a linear regression analysis was performed comparing the simulated quantities to the observed quantities for both Faraday fading and differential mode delay. This analysis is shown 


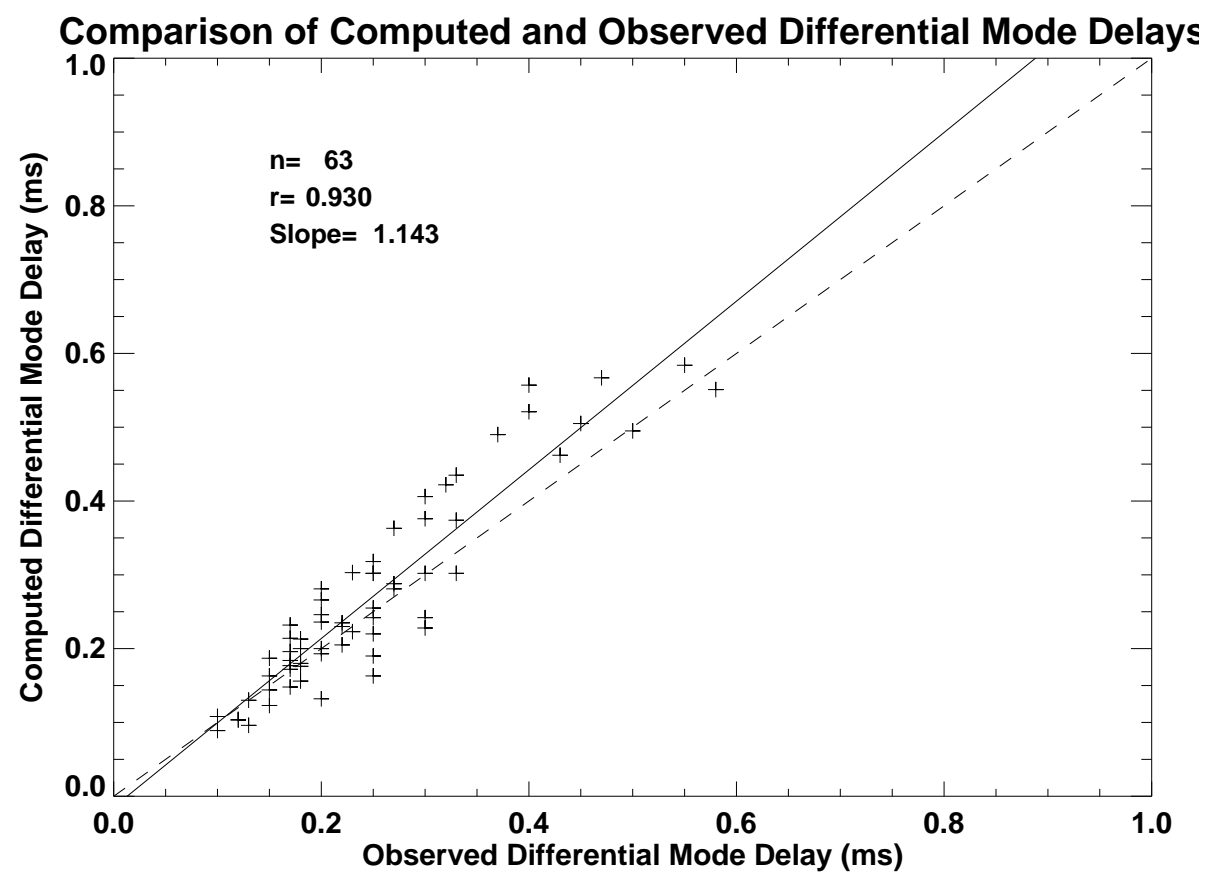

Fig. 6. A linear regression comparison of all simulated and observed differential mode delays for the ISIS II experiment. The dashed line is the ideal fit and the solid line is the best fit. The regression analysis used 63 points and gave a regression coefficient of 0.930 and a slope of 1.14 .

as Fig. 5 for Faraday fading and Fig. 6 for differential mode delay. Plotted are the observed values of either quantity for all passes on the horizontal axis and the corresponding simulated values on the vertical axis. A best fit line is plotted in both cases. For Faraday fading 213 data points were compared, resulting in a best fit slope of 1.07 and a regression coefficient of 0.934 . For mode delay 63 points were compared with a best fit slope of 1.14 and a regression coefficient of 0.930 . Notice that the only group of points that show very poor agreement between observed and computed values is a small cluster of points in Fig. 5. This cluster had an observed fade rate of nearly $7 \mathrm{~Hz}$, but the simulation value was $\sim 3 \mathrm{~Hz}$. The probable reason for this poor agreement is that the $2.5^{\circ}$ latitude spacing between topside soundings was too large spatially in this case as a large change in the ionospheric electron density was observed between the two soundings. Other than this, the comparisons were quite good despite the limitation of not having actual electron density profiles for the ray tracing predictions.

\subsection{Electron density profile considerations}

One of the most interesting features that this study illustrated was that the $f_{o} \mathrm{~F} 2$ peak density is very important in describing the propagation path and characteristics of a transionospheric HF wave. It is not surprising that this is important because the phase difference that accumulates between the $\mathrm{O}$ - and $\mathrm{X}$ modes is directly dependent on electron density. Since both differential mode delay and Faraday fade rate depend on the integrated phase difference between the modes, it was expected that accurately simulating the electron density profile was necessary. The interesting point of this study is that it appears that the IRI model was useful provided the maximum was scaled to the correct $f o \mathrm{~F} 2$ value. Therefore, it appears that the shape of the profiles is of secondary importance. To examine whether this hypothesis is correct a simple test was performed to compare the effects of the profile shapes versus the effects of the $f o \mathrm{~F} 2$ values on simulated wave parameters. A model IRI profile for 1 July 1978, 14:00, at Ottawa, scaled to a peak density of $5.1 \times 10^{11} \mathrm{~m}^{-3}$, was used as a base case. Further IRI profiles of different shapes were used for a variety of ionospheric conditions, with the model constrained to keep the same peak density $\left(5.1 \times 10^{11} \mathrm{~m}^{-3}\right)$ in each case. Profiles were created for three latitudes $\left(0^{\circ}, 45^{\circ}\right.$, and $\left.80^{\circ}\right)$ for both winter (1 January) and summer (1 July) and day (14:00 LT) and night (02:00 LT). This ensured that the profiles would be quite different in shape, but still retained the same peak electron density. Finally, the one base profile was scaled up slightly to create a profile with the same shape as the base profile, but with a peak density of $6.0 \times 10^{11} \mathrm{~m}^{-3}$. The various profiles that were created for this test are listed in Table 1.

Since the goal of this test was to determine which profile parameter, profile shape or $f o \mathrm{~F} 2$ value, is dominant for the ray tracing calculations, a measure of profile shape was 


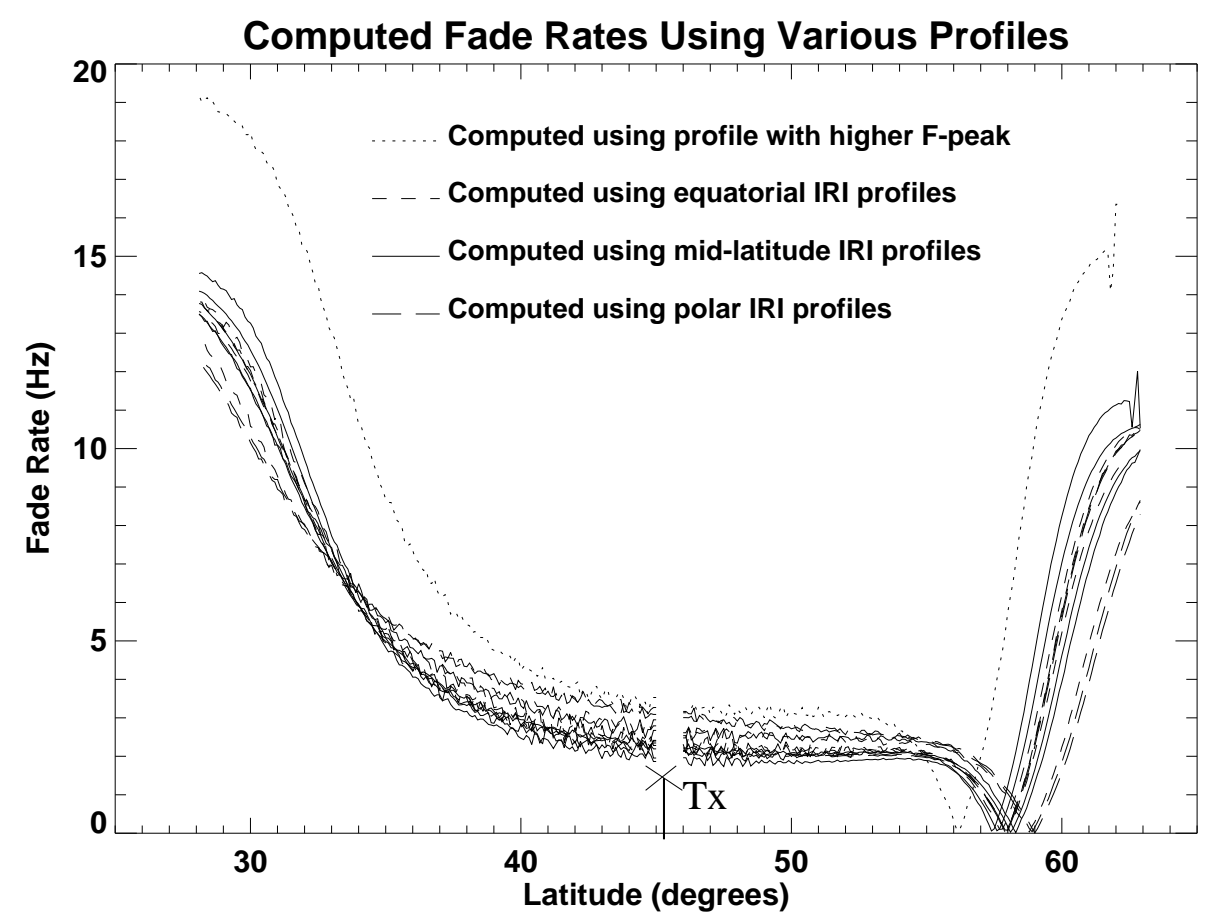

Fig. 7. The simulated Faraday fade rate as a function of satellite latitude using each electron density profile listed in Table 1 . All the results for profiles with the same $f o \mathrm{~F} 2$ value essentially coincide with one another and as such are essentially indistinguishable in the plot. Results show slightly higher fade rate values for profiles with higher TEC above the transmitter. Away from the transmitter, profiles with the same $f_{o} \mathrm{~F} 2$ peak produce similar values, but the profile with a higher peak results in much higher fade rates. Also, notice that the Faraday fade rate values drop to zero around $55^{\circ}$ to $60^{\circ}$ latitude. This is a consequence of the Faraday rotation the satellite observes at this location changing rotation directions. As a result the Faraday fade rate drops to zero as the polarization state switches from rotating in one direction to the other.

required. It was decided to incorporate the Total Electron Content (TEC) value of the various profiles to provide a numerical measure of profile shape. In this study, the TEC value is taken as the integrated electron density from the ground to $1400 \mathrm{~km}$ in units of $10^{16} \mathrm{~m}^{-2}$. There are other measures of profile shape that could have been used, such as $f o \mathrm{~F} 2$ height and width, E-region density, electron density at the satellite location, etc.; however, TEC is a good overall measure of a given profile. Listed in Table 1 is the TEC value for each profile. The TEC value for the scaled up base profile was $22.63 \times 10^{16} \mathrm{~m}^{-3}$ (or $22.63 \mathrm{TEC}$ units). Notice that the profiles in Table 1 have TEC values that range from 14.59 to 27.30 TEC units. Therefore, the profile with a higher $f o \mathrm{~F} 2$ value has a TEC value that falls within the range of the TEC values of the profiles with a lower $f o \mathrm{~F} 2$ value. This allows a study of the effect of $f o \mathrm{~F} 2$ on the ray tracing results to be relatively independent of TEC (or shape) of the profiles.

Each profile was used to predict the signal that would be received by a satellite flying at $1400 \mathrm{~km}$ over a $9.303-\mathrm{MHz}$ transmitter located in Ottawa. The Faraday fade rate and differential mode delay were calculated every $0.1^{\circ}$ latitude. The results of these calculations are presented as Fig. 7 for Faraday fade rate and Fig. 8 for differential mode delay.
At latitudes away from the transmitter, quantities computed for profiles with the same $f o \mathrm{~F} 2$ value showed similar values. Conversely, the profile with a slightly higher $f o \mathrm{~F} 2$ value resulted in much higher fade rate and mode delay values. This indicates that the two received signal parameters are mostly independent of TEC but highly dependent on Fregion peak values at ranges far from the transmitter. Overhead of the transmitter, the two values are observed to be dependent more on TEC than on peak density. The reason for this behaviour is that above the transmitter a radio wave travels essentially straight up and the TEC of a profile dominates the determination of the phase difference that develops between the two modes. When the satellite is north or south of the transmitter, the ray is refractively bent by the highly dense F-region and spends a proportionately longer time in the region in and around the peak density, and the two modes split significantly in this region. This significant splitting accounts for the majority of the accumulated phase difference between the two modes. For example, directly above the transmitter (satellite at $45^{\circ}$ latitude), $\sim 60 \%$ of the total mode delay occurs within $\pm 100 \mathrm{~km}$ in altitude of the $f o \mathrm{~F} 2$ peak, while south of the transmitter with the satellite at $35^{\circ}$ latitude, more than $90 \%$ of the total differential mode delay 


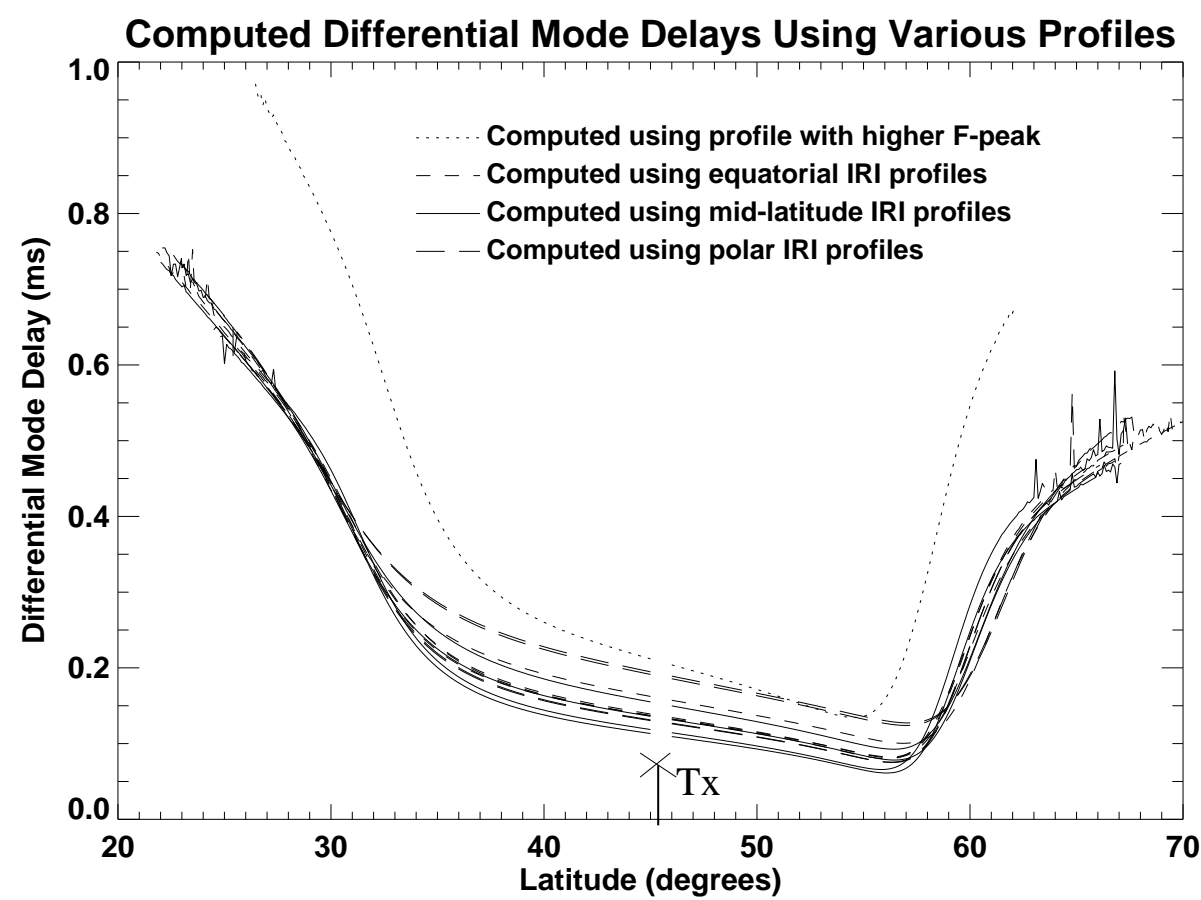

Fig. 8. The simulated differential mode delay at various satellite latitudes using each electron density profile listed in Table 1 . Results show higher differential mode delay values for profiles with higher TEC above the transmitter. Away from the transmitter, profiles with the same $f o \mathrm{~F} 2$ peak produce similar values, but the profile with a higher peak results in much higher differential mode delay values.

Table 1. Various IRI generated electron density profiles. All profiles are generated at $75.7^{\circ} \mathrm{W}$ longitude (Ottawa).

\begin{tabular}{lllll}
\hline $\begin{array}{l}\text { Date } \\
(1978)\end{array}$ & $\begin{array}{l}\text { Time } \\
(\mathrm{LT})\end{array}$ & $\begin{array}{l}\text { Latitude } \\
\left({ }^{\circ} \mathrm{N}\right)\end{array}$ & $\begin{array}{l}\text { TEC } \\
\left(\times 10^{16} \mathrm{~m}^{-2}\right)\end{array}$ & $\begin{array}{l}f o \mathrm{~F} 2 \\
\left(\times 10^{11} \mathrm{~m}^{-3}\right)\end{array}$ \\
\hline 1 Jan & $02: 00$ & 0.0 & 18.23 & 5.1 \\
1 Jan & $14: 00$ & 0.0 & 19.01 & 5.1 \\
1 Jan & $02: 00$ & 45.0 & 14.59 & 5.1 \\
1 Jan & $14: 00$ & 45.0 & 14.75 & 5.1 \\
1 Jan & $02: 00$ & 80.0 & 17.76 & 5.1 \\
1 Jan & $14: 00$ & 80.0 & 17.70 & 5.1 \\
1 July & $02: 00$ & 0.0 & 18.65 & 5.1 \\
1 July & $14: 00$ & 0.0 & 22.28 & 5.1 \\
1 July & $02: 00$ & 45.0 & 18.24 & 5.1 \\
1 July & $14: 00$ & 45.0 & 19.78 & 5.1 \\
1 July & $02: 00$ & 80.0 & 26.72 & 5.1 \\
1 July & $14: 00$ & 80.0 & 27.30 & 5.1 \\
1 July & $14: 00$ & 45.0 & 22.63 & 6.0 \\
\hline
\end{tabular}

occurs within this altitude region. Therefore, this is the reason that, at the frequency of $9.303 \mathrm{MHz}$, the $f_{o} \mathrm{~F} 2$ value and not the shape (or TEC) of the electron density profile is critical in obtaining reasonable ray tracing results for the experiment presented in this study.

\section{Conclusions}

Data from the ISIS II satellite mission has been analyzed in preparation for the upcoming ePOP mission. The received signal data from the ISIS II satellite mission showed two prominent features: differential mode delay and periodic Faraday fading. Both features were observed to the south of the transmitter where nearly equal O- and X-mode amplitudes allowed clear Faraday fading and higher electron densities resulted in larger mode splitting. The rate of measured periodic fading varied up to $13 \mathrm{~Hz}$ and the magnitude of differential mode delay was as high as $0.8 \mathrm{~ms}$. Ray tracing predicted the observed fade rates and differential mode delays using electron densities derived from measured $f o \mathrm{~F} 2$ values and IRI profiles. The simulated and observed data showed very similar trends for each of the examined passes. This good agreement was dependent on having a representative electron density profile for the ray tracing analysis. The two parameters which were used to characterize an electron density profile were the profile shape (examined using TEC values) and the peak electron density ( $f o \mathrm{~F} 2)$. The comparison between the ISIS II measurements and the computations indicated that the $f o \mathrm{~F} 2$ parameter, and not the TEC parameter, essentially determined the outcome of the simulations. This behaviour is a consequence of refraction causing the wave to travel longer near the peak density where larger splitting of the modes due to the higher electron densities in this 
region occurs. This was clearly the case for the transmitter frequency of $9.303 \mathrm{MHz}$, which was a few $\mathrm{MHz}$ above $f o \mathrm{~F} 2$. Of course, the use of higher transmitter frequencies would cause the wave to be refracted less and the $f o \mathrm{~F} 2$ value would likely not dominate the received signal parameters to such an extent.

This paper has demonstrated that ray tracing computations can reasonably reproduce transionospheric propagation characteristics under quiet geomagnetic conditions and at midlatitudes (i.e., south of the Ottawa transmitter in this study). No evidence of Faraday fading or differential mode delay was observable north of the Ottawa transmitter for the ISIS II study. As discussed, previous results (James et al., 2006; James, 2005) indicated that the X-mode to O-mode ratio was on the order of $10 \mathrm{~dB}$ in this region and thus Faraday rotation and clear mode splitting were not expected. Instead of a simple linear wave, the resulting polarization state would be much more complex under these conditions. The RRI instrument on the ePOP satellite will be much more sensitive and consist of two crossed dipoles; therefore, it may be possible to interpret these complex signals.

Acknowledgements. We acknowledge the support of the Canadian Space Agency and the Natural Sciences and Engineering Research Council (NSERC) to the CASSIOPE/ePOP project for our research. International Reference Ionosphere (IRI) electron density profiles have been provided by the Space Physics Data Facility (SPDF). Digitized online ISIS II data has been supplied by R. F. Benson at the National Aeronautics and Space Administration (NASA) Goddard Space Flight Center (GSFC) and Coordinated Data Analysis Web (CDAWeb).

Topical Editor M. Pinnock thanks W. Bristow and A. Stocker for their help in evaluating this paper.

\section{References}

Bilitza, D., Huang, X., Reinisch, B. W., Benson, R. F., Hills, H. K., and Schar, W. B.: Topside Ionogram Scaler With True Height Algorithm (TOPIST): Automated processing of ISIS topside ionograms, Radio Sci., 39, RS1S27, doi:10.1029/2002RS002840, 2004.

Bilitza, D.: International Reference Ionosphere 2000, Radio Sci., 36, 261-275, 2001.

Budden, K. G.: Radio Waves in the Ionosphere, Cambridge University Press, 1961.

Daniels, F.: ISIS-II Spacecraft, CRC Report No. 1218, Communications Research Centre, Ottawa, 1971.

Franklin, C. A. and Maclean, M. A.: The Design of SweptFrequency Topside Sounders, Proceeding of the IEEE, 57(6), 897-929, 1969.
Gillies, R. G.: Modelling of Transionospheric HF Radio Wave Propagation for the ISIS II and ePOP Satellites, M.Sc. Thesis, Institute of Space and Atmospheric Studies, University of Saskatchewan, 2006.

Greenwald, R. A., Baker, K. B., Dudeney, J. R., Pinnock, M., Jones, T. B., Thomas, E. C., Villain, J.-P., Cerisier, J. C., Senior, C., Hanuise, C., Hunsucker, R. D., Sofko, G., Koehler, J., Nielsen, E., Pellinen, R., Walker, A. D. M., Sato, N., and Yamagishi, H.: DARN/SuperDARN: A Global View of the Dynamics of HighLatitude Convection, Space Sci. Rev., 71, 761-796, 1995.

Haselgrove, J.: The Hamiltonian ray path equations, J. Atmos. Terr. Phys., 25, 397-399, 1963.

James, H. G.: Effects on transionospheric HF propagation observed by ISIS at middle and auroral latitudes, Adv. Space Res., 38(11), 2303-2312, 2006a.

James, H. G.: A digital radio receiver for ionospheric research, NATO Specialist Meeting IST-056, "Characterizing the Ionosphere”, Paper 23, Fairbanks, AK, USA, 12-16 June 2006b.

James, H. G.: High-frequency direction finding in space, Review of Scientific Instruments, 74(7), 3478-3486, 2003.

James, H. G., Gillies, R. G., Hussey, G. C., and Prikryl, P.: HF fades caused by multiple wave fronts detected by a dipole antenna in the ionosphere, Radio Sci., 41, RS4018, doi:10.1029/2005RS003385, 2006.

Maus, S., Macmillan, S., Chernova, T., Choi, S., Dater, D., Golovkov, V., Lesur, V., F. Lowes, Luhr, H., Mai, W., McLean, S., Olsen, N., Rother, M., Sabaka, T., Thomson, A., and Zvereva, T.: The 10th generation international geomagnetic reference field, Physics of the Earth and Planetary Interiors, 151, 320-322, 2005.

MacDougall, J. W., Grant, I. F., and Shen, X.: The Canadian Advanced Digital Ionosonde: Design and Results, Rep. UAG-104, Ionosondes and Ionosonde Networks, 21-27, World Data Center A, Boulder, 1995.

NSSDC: ISIS/Alouette topside sounder data restoration project, available from: http://nssdc.nasa.gov/space/isis/isis-status.html, 2004.

Wang, L., MacDougall, J. W., and James, H. G.: Ionospheric structure effects on $\mathrm{HF}$ radio wave propagation for the Enhanced Polar Outflow Probe (e-POP) satellite mission, Radio Sci., 39, RS2019, doi:10.1029/2003RS002975, 2004.

Yau, A. W, James, H. G., and Lui, W.: The Canadian Enhanced Polar Outflow Probe (ePOP) mission in ILWS, Adv. Space Res., 38(8), 1870-1877, 2006.

Yau, A. W., Cogger, L. L., King, E. P., Knudsen, D. J., Murphree, J. S., Trondsen, T. S., Tsuruda, K., James, H. G., and Walkty, I.: The Polar Outflow Probe (POP): Science Objectives and Instrument Development, Canadian Aeronautics and Space Journal, 48(1), 39-49, 2002. 